\title{
Fragmentation and the Bose-glass phase transition of the disordered one-dimensional Bose gas
}

\author{
Luca Fontanesi, Michiel Wouters, and Vincenzo Savona \\ Institute of Theoretical Physics, Ecole Polytechnique Fédérale de Lausanne EPFL, CH-1015 Lausanne, Switzerland
}

(Received 15 October 2010; revised manuscript received 8 December 2010; published 28 March 2011)

\begin{abstract}
We investigate the superfluid-insulator quantum phase transition in a disordered one-dimensional Bose gas in the mean-field limit by studying the probability distribution of the density. The superfluid phase is characterized by a vanishing probability to have zero density, whereas a nonzero probability marks the insulator phase. This relation is derived analytically and confirmed by a numerical study. This fragmentation criterion is particularly suited for detecting the phase transition in experiments. When a harmonic trap is included, the transition to the insulating phase can be extracted from the statistics of the local density distribution.
\end{abstract}

DOI: 10.1103/PhysRevA.83.033626

PACS number(s): $03.75 . \mathrm{Hh}, 64.70 . \mathrm{Tg}, 67.85 . \mathrm{Bc}$

\section{INTRODUCTION}

A disordered low-dimensional noninteracting system is expected to always be in an insulating state [1]. Manybody interactions, in the case of Bose particles, may induce a phase transition to a superfluid state. This transition is conventionally defined through the superfluid fraction or by the long-range behavior of the one-body density matrix [2]. In one dimension at zero temperature, the latter is expected to decay algebraically in the superfluid (SF) phase and exponentially in the Bose-glass (BG) phase. The versatility and tunability of ultracold atomic systems have motivated, in recent years, the study of this fundamental phenomenon in a low-dimensional disordered Bose gas [3-9], for which, however, neither the superfluid fraction nor the one-body density matrix are easily accessible in experiments. Several different ways of characterizing this phase transition have thus recently been discussed [10-14]. Despite the many experimental attempts [5,15-17], however, clear evidence of the superfluid-insulator transition in one-dimensional (1D) Bose gases has not yet been obtained.

The notion of fragmentation has been widely used in relation to Bose-Einstein condensation [18,19]. In the case of a disordered Bose gas, fragmentation has been frequently evoked as a criterion for the transition from superfluid to Boseglass phase $[5,19]$. To our knowledge, however, a rigorous definition of fragmentation of the density profile and proof of its relation to the quantum phase of the gas are still lacking.

\section{FRAGMENTATION CRITERION}

A quantity that naturally characterizes fragmentation is the probability distribution of the density (PDD), i.e., $P\left(\rho_{0}\right)$, in the limit $\rho_{0} \rightarrow 0$. We define a state to be fragmented if $P\left(\rho_{0} \rightarrow 0\right)$ is nonzero. In this article we show that in the weakly interacting regime the density distribution fragments at the transition from superfluid to Bose glass. This result bears a clear experimental advantage, as the density profile of a Bose gas is a quantity that can be easily investigated, within the spatial resolution of the experimental apparatus. To make a link to current experiments, a realistic situation of a gas confined in a harmonic trap is examined [20]. In this case, the transition between a quasicondensate and an insulator - and a possible spatial separation between the two phases-can be unveiled through a local investigation of the statistical distribution of the density profile.

To establish a link between the PDD and the phase transition, we study the superfluid fraction of the Bose gas. This quantity can be characterized by evaluating the response of the system to a velocity field, which is equivalent to imposing twisted boundary conditions [21]. In fact, the superfluid fraction is proportional to the difference of the energies in the moving frame, $E_{\Theta}$, and in the rest frame, $E_{0}$, as [22]

$$
f_{S}=\frac{2 m L^{2}}{\hbar^{2} N} \lim _{\Theta \rightarrow 0} \frac{E_{\Theta}-E_{0}}{\Theta^{2}} .
$$

Here $\Theta$ is the total phase twist, $m$ is the mass of the bosons, $L$ is the length of the system, and $N$ is the number of particles. A mean-field model of the weakly interacting 1D Bose gas requires a description in terms of a density, $\hat{\rho}=\rho_{0}+\delta \hat{\rho}$, and a phase operator, $\hat{\theta}$, as $\hat{\Psi}(r) \simeq e^{i \hat{\theta}(x)} \sqrt{\hat{\rho}(x)}$. The ground-state density is the solution of a Gross-Pitaevskii equation (GPE) [23],

$$
\left[-\hbar^{2} \partial_{x}^{2} /(2 m)+V(x)+g \rho_{0}(x)\right] \sqrt{\rho_{0}(x)}=\mu \sqrt{\rho_{0}(x)}
$$

where $\mu$ is the chemical potential, $V(x)$ is the external potential, and $g$ is the interaction constant. The validity of the Bogoliubov prescription, $\langle\delta \hat{\rho}\rangle / \rho_{0} \ll 1$, ensures that $\rho_{0}$ contains the relevant information about the density distribution. In fact, the Bogoliubov approximation becomes more and more accurate for increasing average density, $N_{0} / L$, at constant interaction $g N_{0} / L$, with $N_{0}$ being the number of bosons in the ground state. Deep in the mean-field limit $N_{0} / L \rightarrow \infty$, the relative contribution of the fluctuations $\langle\delta \hat{\rho}\rangle / \rho_{0} \propto 1 / \rho_{0} \rightarrow 0$ and the density profile is completely described by $\rho_{0}(x)$ [24]. From the Gross-Pitaevskii energy functional, computed at leading order in the phase twist, the energy difference is given by the kinetic term

\footnotetext{
*1uca.fontanesi@epfl.ch
}

$$
E_{\Theta}-E_{0}=\int \frac{[\nabla \theta(x)]^{2}}{2 m} \rho_{0}(x) d x
$$


where $\theta=\langle\hat{\theta}\rangle$. Minimizing this energy with the constraint $\int \nabla \theta=\Theta$ shows that the total superfluid fraction is related to the harmonic average of the density $[12,25]$ as

$$
\frac{1}{\rho_{S}}=\int \frac{1}{\rho_{0}(x)} d x=\int \frac{1}{\rho_{0}} P\left(\rho_{0}\right) d \rho_{0} .
$$

The convergence of the integral in Eq. (4) is determined by the behavior of $P\left(\rho_{0}\right)$ in the limit $\rho_{0} \rightarrow 0$. If we express $P\left(\rho_{0} \rightarrow 0\right)=\rho_{0}^{\beta}$, then the condition to be in the superfluid phase is $\beta>0$. A nonzero value of $P(0)$, on the contrary, implies the insulator phase.

\section{NUMERICAL SIMULATIONS}

We compute the PDD numerically, by solving Eq. (2) on finite size systems with periodic boundary conditions. Configuration average has been adopted to increase the precision of the statistical sampling. This analysis is done for Gauss-distributed and Gauss-correlated disorder, described by

$$
\left\langle V(x) V\left(x^{\prime}\right)\right\rangle=\Delta_{g}^{2} e^{-\frac{\left(x-x^{\prime}\right)^{2}}{2 \eta_{s}^{2}}},
$$

where $\Delta_{g}$ is the disorder amplitude and $\eta_{g}$ is the spatial correlation length that introduces an additional energy scale, $E_{c}=\frac{\hbar^{2}}{2 m \eta^{2}}$. The subscript " $g$ " denotes a disordered Gaussian potential, while " $s$ " will be used later on to denote a speckle potential. The third energy entering the problem is the interaction energy, $U=g N_{0} / L$.

Previously we have characterized the phase boundary in independent ways, through the study of the superfluid fraction and the one-body density matrix $[11,12]$. Two limiting cases have been identified [12]: a Thomas-Fermi regime (TF), where $E_{c} \ll U$, and a white-noise limit (WN), marked by $E_{c} \gg$ $U$. In these regimes, the phase boundary obeys power-law relations, $\Delta / E_{c}=C\left(U / E_{c}\right)^{\gamma}$, with $\gamma$ respectively equal to 1 and $3 / 4$ (cf. Fig. 5).

Figure 1(a) shows the PDD for fixed $\Delta_{g}=12.8 E_{c}$ and increasing interaction $U=25.6 E_{c}, 33.28 E_{c}$, $38.4 E_{c}$, and $46.08 E_{c}$ (all in the $\mathrm{TF}$ regime). From our previous analysis $[11,12]$ these four values correspond to the BG (two lowest), phase boundary, and SF phases, respectively. In the homogeneous case $P\left(\rho_{0}\right)$ is expected to have a single peak at the value $\rho_{0} / \rho_{H}=1$, where $\rho_{H}$ is the constant solution of the homogeneous problem. The inclusion of a small disorder [26] broadens this peak, but $P\left(\rho_{0}\right)$ preserves a vanishing tail for $\rho_{0} \rightarrow 0$ [solid blue curve in Fig. 1(a)] in the superfluid phase. For decreasing interaction the weight of the low-density part becomes more important [green dashed curve in Fig. 1(a)] until the phase boundary is eventually crossed and the PDD develops a finite component in the limit $\rho_{0} \rightarrow 0$ [red dot-dashed lines in Fig. 1(a)]. Figure 1(b) shows a similar analysis carried out for the WN regime, for $\Delta_{g}=0.016 E_{c}$ and $U=0.0032 E_{c}, 0.004 E_{c}, 0.0048 E_{c}$, and $0.0064 E_{c}$. As for the TF case, from our previous study these four values lie in the BG (two lowest), phase boundary, and SF phases, respectively.

Comparing Figs. 1(a) and 1(b), it is clear that the PDD has different shapes in the TF and WN regimes. However, in both cases, the fragmentation allows one to differentiate between the SF and BG phases. The numerical analysis summarized in
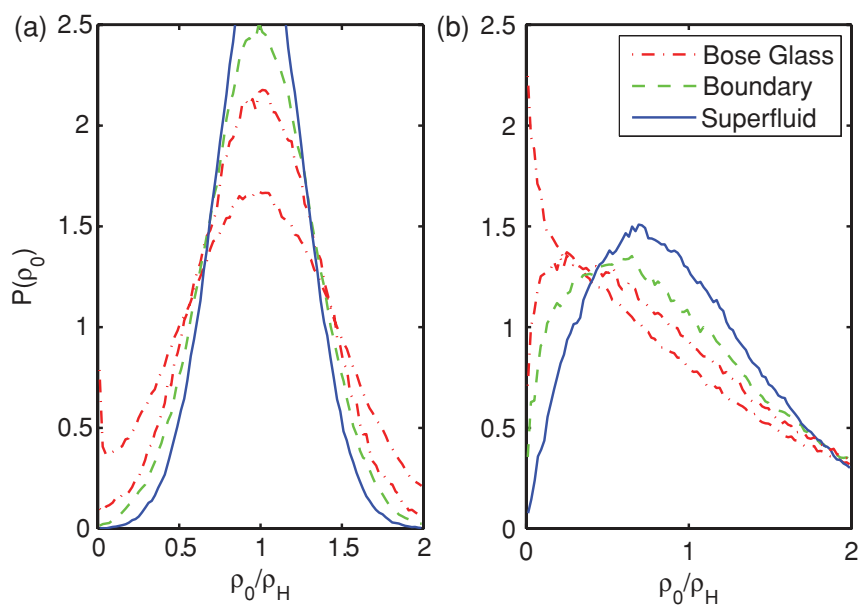

FIG. 1. (Color online) Probability distribution of the density in two different regimes in the presence of Gaussian disordered potential. (a) Toward the TF regime, $\Delta_{g}=$ $12.8 E_{c}$ for $U=25.6 E_{c}, 33.28 E_{c}$ (red dot-dashed), $38.4 E_{c}$ (green dashed), and $46 E_{c}$ (blue solid). (b) Toward the WN limit, $\Delta_{g}=$ $0.016 E_{c}$ for $U=0.0032 E_{c}, 0.004 E_{c}$ (red dot-dashed), $0.0048 E_{c}$ (green dashed), and $0.0064 E_{c}$ (blue solid). $\rho_{H}$ is the density in the homogeneous case.

Fig. 1 confirms the criterion stemming from Eq. (4), namely, that the SF fraction is nonzero if and only if $P\left(\rho_{0} \rightarrow 0\right)=0$.

$P\left(\rho_{0}\right)$ can be evaluated analytically in certain regimes. In the TF regime, when the kinetic term is negligible, the density follows the external potential according to the TF approximation as

$$
\begin{array}{ccc}
\rho_{0}(x)=[\mu-V(x)] / g & \text { if } & V(x)<\mu, \\
\rho_{0}(x)=0 & \text { if } & V(x)>\mu .
\end{array}
$$

In this regime the distribution of $\rho_{0}(x)$ reproduces the distribution of the potential at any finite value, with an additional finite contribution at zero, given by the sum of the regions where $V(x)>\mu$. A very similar feature can be indeed noticed in the insulating case of Fig. 1(a) (dot-dashed line) where the PPD has a Gaussian-like shape with a peaked contribution in zero. Following the fragmentation argument, this case is always insulating as it can be expected since the absence of a kinetic component prevents the formation of any quasi-long-range order or superfluid flow. This is not in contradiction with the phase transition found for large values of $U / E_{c}$, because the kinetic energy corrections to Eq. (6) are responsible for the buildup of the quasi-long-range order.

We perform the same analysis in the presence of a speckle potential. This disorder results from the interference pattern produced by the scattering of coherent light through a rough plate and is currently used in experiments on ultracold atomic gases $[3,16,20,27]$. Its amplitude, $\Delta_{s}$, is defined as the standard deviation of the potential and its energy distribution is bound from one side (above or below depending on the detuning of the laser) and decays exponentially on the opposite side [28]. In accordance with the experimental realization, the speckle 
potential is generated starting from a field, in real space, with a uniformly distributed random phase,

$$
E(x)=\sqrt{\frac{I \pi}{k_{c} \mathcal{L}}} \sum_{k=-k_{c}}^{k_{c}} e^{i k x} e^{i \theta(k)},
$$

where $I$ is the intensity of the impinging light, $\mathcal{L}$ is the length of the scattering plate that gives the quantization in $k$ space, $\Delta k=$ $2 \pi / \mathcal{L}, \theta(k)$ are the random phases in the interval $(-\pi, \pi]$, the distribution in real space is defined on a 1D segment, and the sum is extended to each point of the grid. The window, of width $k_{c}$, is applied on the distribution in Fourier space to obtain the distribution of the disorder as the square of the resulting field in real space. Its correlation length $\eta_{s}$ is given by the numerical aperture of the focusing lens and in particular is linked to the cutoff in $k$-space as $\eta_{s}=1 / k_{c}$. In the simulations that follow, $\eta_{s}$ is taken as the unit of length, consequently $k_{c}$ is the unit in Fourier space, and $\mathcal{L}$ is $200 \eta_{s}$.

The fragmentation study, shown in Fig. 2, is performed for fixed disorder amplitude, $\Delta_{s}=3.2 E_{c}$, for increasing interaction energy, $U=2.6,3.1$, and $3.5 E_{c}$. As we are considering a speckle potential bound from above (attractive), the distribution is not centered around its mean value, so the choice of the potential strongly affects the shape of the PDD, but, as we have checked, the limiting behavior $P\left(\rho_{0} \rightarrow 0\right)$ is only determined by the phase of the gas, consistently with the conclusions drawn for the Gaussian disorder. From this inspection we extract the critical value of the interaction energy $U \simeq 3.1 E_{c}$, which will be important in the analysis that follows.

\section{TRAPPED CASE}

Homogeneous systems are useful theoretical tools to inspect the phase transition, but current experiments $[5,16]$ are performed on trapped systems. The harmonic trap introduces a spatial inhomogeneity in the interaction energy $U(x)=$ $g \rho_{0}(x)$. To deal with a realistic situation we consider a speckle disorder on the top of a harmonic trapping potential, as it has been investigated in recent experiments [16,27], and

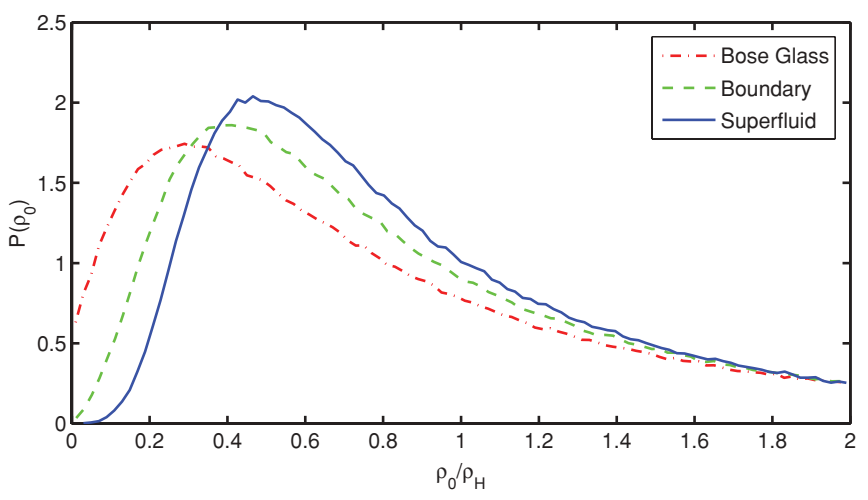

FIG. 2. (Color online) Probability distribution of the density in the presence of attractive speckle potential, obtained from an average on disorder configurations. Three values of interaction across the phase boundary are shown, $U=2.6 E_{c}$ (red dot-dashed), 3.1 $E_{c}$ (green dashed), and $3.5 E_{c}$ (blue solid), for fixed disorder strength $\Delta_{s}=$ $3.2 E_{c} . \rho_{H}$ is a constant value, the density in the homogeneous case. we perform the simulations on a system of experimentally achievable size [20] $\left(200 \eta_{s}\right)$. We stress that the PDD can be obtained by evaluating the histogram of the density at a fixed position for several disorder realizations. In what follows, we represent these histograms as $P_{x}(U)$, namely, as a function of the interaction energy, $U=g \rho_{0}$, instead of the density $\rho_{0}$. In this way the average of $P_{x}(U)$ is the average interaction energy and can be directly associated with a point on the phase diagram.

The density plots in Fig. 3 show the spatially resolved probability distributions, $P_{x}(U)$, as a function of position and interaction energy for fixed disorder amplitude, $\Delta_{s}=3.2 E_{c}$. The density is expressed in terms of the interaction energy $U(x)=g \rho_{0}(x)$, that is, the quantity that determines the phase transition in the mean-field limit. The blue dashed line is the average interaction energy $\bar{U}(x)=\int g \rho_{0}(x) P_{x}\left(\rho_{0}\right) d \rho_{0}$, and the red solid line represents the interaction profile in the absence of disorder $U_{C}(x)$, i.e., the solution of the GPE with $V(x)=m \omega_{t}^{2} x^{2} / 2$, where $\omega_{t}$ is the trapping frequency. Note that the disorder combined with a smooth trapping potential makes the average density profile different from the clean one; in fact it extends beyond the disorderless $\mathrm{TF}$ radius and it reaches a lower value in the center of the trap. This fact is relevant when comparing the critical values of interaction at the transition between the trapped and untrapped cases.
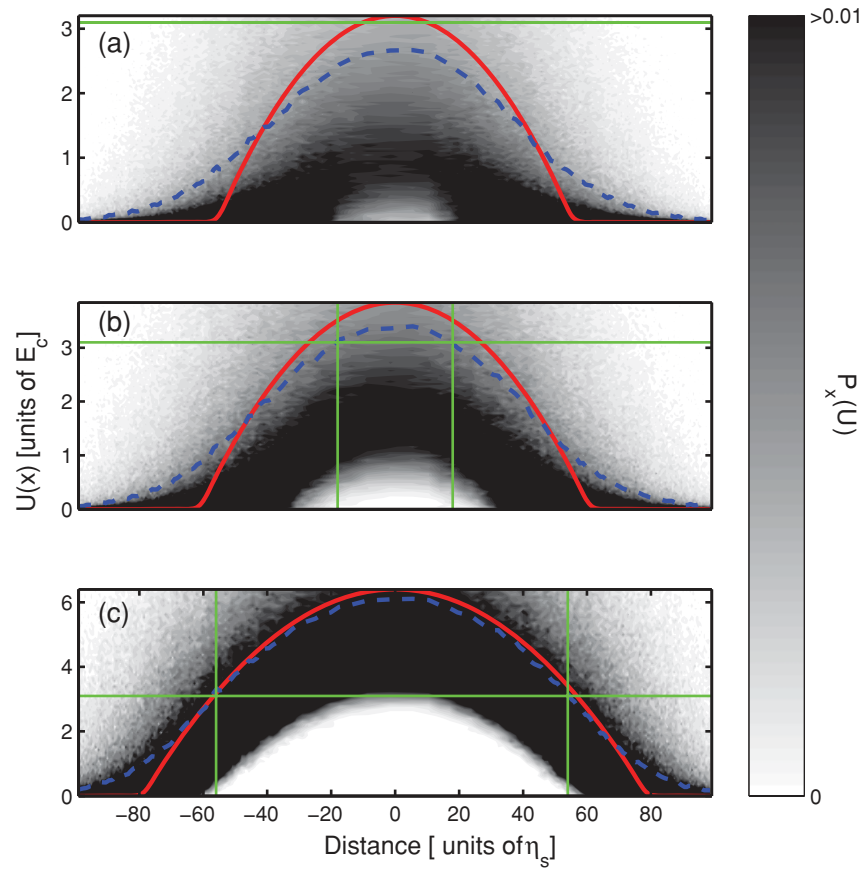

FIG. 3. (Color online) Local probability distribution of the interaction energy (proportional to the density) in a harmonic trap $\left(\hbar \omega_{t}=0.064 E_{c}\right.$ ) for three different values of the interaction energy $U_{C}(0)$ : (a) $3.2 E_{c}$, (b) $3.84 E_{c}$, and (c) $6.4 E_{c}$, and for fixed speckle disorder intensity $\left(\Delta_{s}=3.2 E_{c}\right)$. The distributions are obtained by averaging over 2000 configurations of disorder. The red solid lines are the interaction energy profiles in the disorderless cases. Blue dashed lines represent the spatial distribution of the average interaction energies. The green thin lines indicate the boundary in the thermodynamic limit extracted analyzing the untrapped case. 
It is important to understand whether the fragmentation threshold, in the trapped case, coincides with the one extracted in the thermodynamic limit. For the chosen value of the disorder amplitude, the fragmentation analysis in the untrapped case gives a transition at an interaction strength of $U_{H} \simeq 3.1 E_{c}$ (cf. Fig. 2). In Fig. 3(a) the gas is fragmented at all positions, consistent with the homogeneous boundary, as the maximal interaction energy $U(0) \simeq 2.7 E_{c}<U_{H}$. When the interaction energy is increased, SF and BG phases coexist [see Figs. 3(b) and 3(c)]. The fragmentation threshold of the interaction energy can be extracted directly from the spatially resolved PDDs; these values for the average interaction at the threshold coincide with $U_{H}$ [cf. Figs. 3(b) and 3(c)]. This is true as long as the local density approximation is valid $\left(\hbar \omega_{t} \ll U\right)$. For tighter traps (simulations not shown), the fragmentation line moves to lower values of the average interaction energy, which can be interpreted as a penetration of the SF into the insulating state (cf. Fig. 5). This is obvious when considering the limit $\hbar \omega_{t} \gg U, \Delta$, where the density profile is the nonfragmented harmonic oscillator ground state. The penetration effect is more pronounced when the transition occurs at the edges of the trap where the density gradient is steeper. Therefore, the best agreement between the phase transition of the trapped and homogeneous gases is obtained when the transition occurs close to the center of the trap.

\section{FINITE RESOLUTION}

In current experiments the resolution of the apparatus represents the main limitation to the correct interpretation of the experimental data. For this purpose we study the role of a finite resolution on the statistical analysis that we propose in this article. The resolution is included in the simulation via a convolution of the ground-state wave function with a Gaussian of standard deviation $R$. The finite resolution makes it harder to identify the insulator phase through a statistical study of the density because it smooths out the profile, cutting narrow minima from the statistics. This limitation acts differently in different regimes: supposing the ratio $\eta / R$ to be fixed, the finite resolution would not affect the statistics in the WN limit, where modulations of the density occur on the scale of the healing length, much longer than the resolution. On the contrary, if $\eta / R \lesssim 1$, this would strongly affect the statistics in the TF regime, where the length scale of the modulation would be the typical correlation length of the potential. Figure 4 shows the effect of finite resolution on the probability distribution of a trapped Bose gas for $U_{C}(0)=3.2 E_{c}$ and $\Delta_{s}=3.2 E_{c}$. The statistical analysis of the distribution [Fig. 3(a)] identifies a completely fragmented state. The introduction of a finite resolution $(R=1)$ in Fig. 4(a) opens a window in the center of the trap as if the Bose gas were partially superfluid. This effect becomes even more significant for a worse resolution $(R=2)$, as shown in Fig. 4(b), where the gas appears to be predominantly superfluid. For this set of parameters, the transition would be placed at $U \sim 3$ and $2.9 E_{c}$, respectively. Therefore, a reasonable criterion to correctly estimate the fragmentation threshold is $R \lesssim \sqrt{\eta^{2}\left(1+E_{c}^{2} / \Delta^{2}\right)+\xi^{2}}$, where all the relevant length scales are taken into account.

Both the finite resolution and the harmonic trap tend to displace the apparent fragmentation threshold toward the

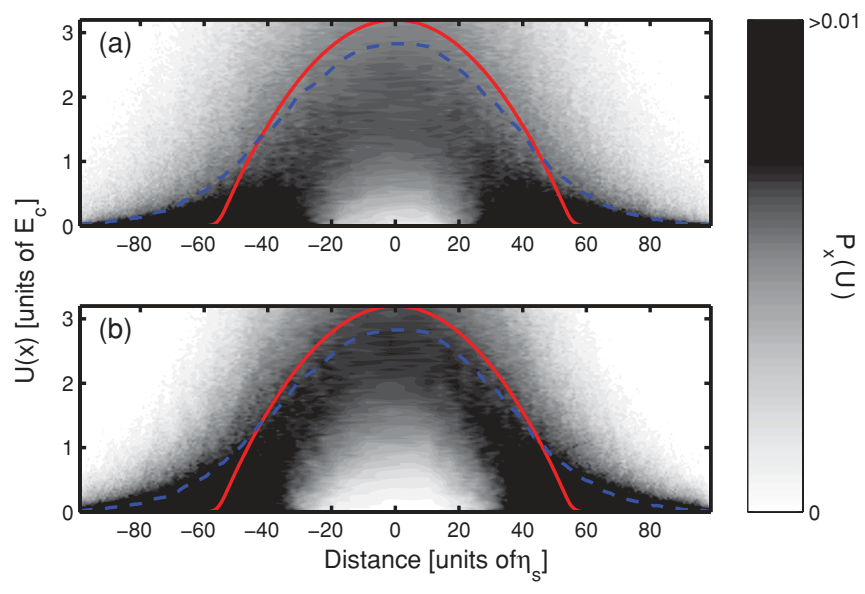

FIG. 4. (Color online) Effect of a finite resolution on the local probability distribution of the interaction energy in a harmonic trap $\left(\hbar \omega_{t}=0.064 E_{c}\right.$ ) for the insulating state of Fig. 3(a): $U_{C}(0)=$ $3.2 E_{c}$ and $\Delta_{s}=3.2 E_{c}$. Panel (a) shows a resolution $R=1$, whereas in panel (b) $R=2$.

insulator phase with respect to the actual boundary. Therefore, in experiments, the superfluid fraction is overestimated, but a quantitative correction to the boundary can be computed knowing the value of the resolution and the intensity of the trap. A qualitative sketch of the effect of real conditions on the actual phase diagram is shown in Fig. 5. From this sketch it

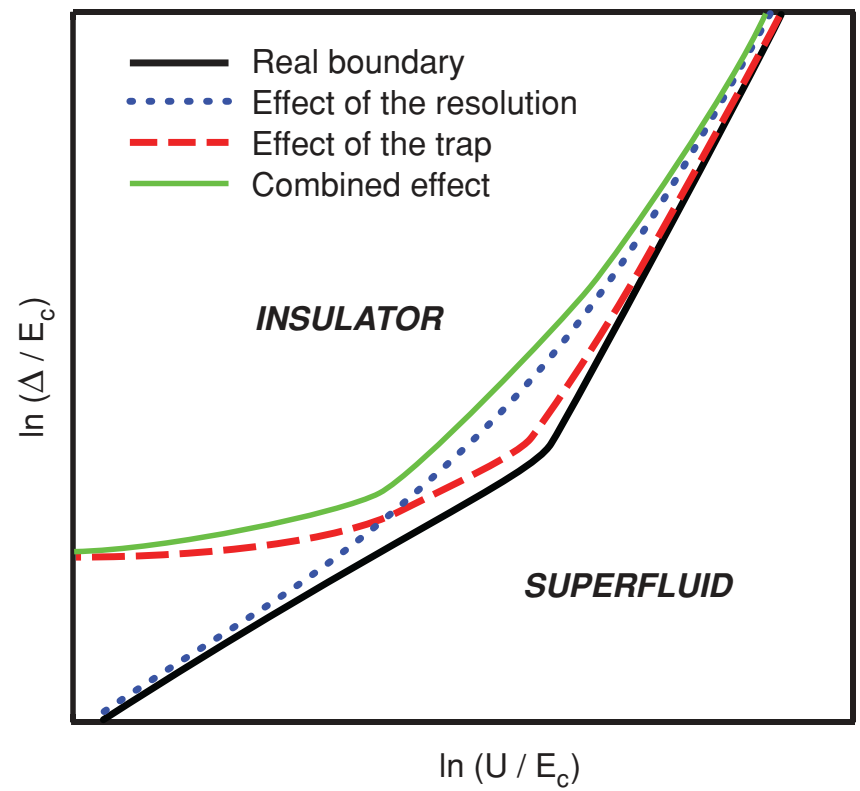

FIG. 5. (Color online) Sketch of the phase diagram of the 1D Bose gas in the presence of disorder. The effect of realistic limitations in experiments are qualitatively shown. The black thick line represents the actual phase boundary, and the blue dotted line is the phase boundary extracted with a finite resolution of the experimental setup, for a fixed ratio $\eta / R$ (see text). The red dashed line is the fragmentation boundary considering a harmonic potential of fixed trapping frequency. The trap tends to displace the boudary toward the insulating phase. For $\hbar \omega_{t} \gtrsim U$ the local density approximation breaks down and the profile is never fragmented. The combined effect is shown by the green thin line. 
appears that the trapped system no longer reflects the properties of the thermodynamic limit deep in the WN regime. Indeed, fragmentation would occur on a scale much longer than the ground state of the trap.

\section{CONCLUSIONS}

We have demonstrated a relation between the quantum phase transition of the 1D Bose gas and the probability distribution of the density in the mean-field limit. We found that the superfluid phase is marked by a vanishing probability at zero density whereas the insulating phase develops a finite component in the limit $P\left(\rho_{0} \rightarrow 0\right)$. This gives a viable route to the determination of the phase of the gas, and therefore of the phase transition, through a statistical analysis of the density profiles.

\section{ACKNOWLEDGMENTS}

We thank B. Deissler, R. Hulet, P. Lugan, E. Runge, and L. Sanchez-Palencia for stimulating discussions. This research has been supported by the Swiss National Science Foundation through Project No. 200021-117919.
[1] E. Abrahams, P. W. Anderson, D. C. Licciardello, and T. V. Ramakrishnan, Phys. Rev. Lett. 42, 673 (1979).

[2] M. P. A. Fisher, P. B. Weichman, G. Grinstein, and D. S. Fisher, Phys. Rev. B 40, 546 (1989).

[3] J. Billy et al., Nature (London) 453, 891 (2008).

[4] G. Roati et al., Nature (London) 453, 895 (2008).

[5] B. Deissler et al., Nat. Phys. 6, 354 (2010).

[6] M. Pasienski et al., Nat. Phys. 6, 677 (2010).

[7] B. Deissler, E. Lucioni, M Modugno, G. Roati, L. Tanzi, M. Zaccanti, M. Inguscio, and G. Mudugno, New J. Phys. 13, 023020 (2011).

[8] G. Modugno, Rep. Prog. Phys. 73, 102401 (2010).

[9] L. Sanchez-Palencia and M. Lewenstein, Nat. Phys. 6, 87 (2010).

[10] B. Damski, J. Zakrzewski, L. Santos, P. Zoller, and M. Lewenstein, Phys. Rev. Lett. 91, 080403 (2003).

[11] L. Fontanesi, M. Wouters, and V. Savona, Phys. Rev. Lett. 103, 030403 (2009).

[12] L. Fontanesi, M. Wouters, and V. Savona, Phys. Rev. A 81, 053603 (2010).

[13] D. Delande and J. Zakrzewski, Phys. Rev. Lett. 102, 085301 (2009).

[14] J. Carrasquilla, F. Becca, A. Trombettoni, and M. Fabrizio, Phys. Rev. B 81, 195129 (2010).

[15] L. Fallani, J. E. Lye, V. Guarrera, C. Fort, and M. Inguscio, Phys. Rev. Lett. 98, 130404 (2007).
[16] Y. P. Chen, J. Hitchcock, D. Dries, M. Junker, C. Welford, and R. G. Hulet, Phys. Rev. A 77, 033632 (2008).

[17] M. White, M. Pasienski, D. McKay, S. Q. Zhou, D. Ceperley, and B. DeMarco, Phys. Rev. Lett. 102, 055301 (2009).

[18] P. Nozières, in Bose-Einstein Condensation, edited by A. Griffin, D. W. Snoke, and S. Stingari (Cambridge University Press, Cambridge, UK, 1995), Chap. 2.

[19] P. Lugan, D. Clement, P. Bouyer, A. Aspect, M. Lewenstein, and L. Sanchez-Palencia, Phys. Rev. Lett. 98, 170403(2007).

[20] D. Dries, S. E. Pollack, J. M. Hitchcock, and R. G. Hulet, Phys. Rev. A 82, 033603 (2010).

[21] E. H. Lieb, R. Seiringer, and J. Yngvason, Phys. Rev. B 66, 134529 (2002).

[22] M. E. Fisher et al., Phys. Rev. A 8, 1111 (1973).

[23] C. Mora and Y. Castin, Phys. Rev. A 67, 053615 (2003).

[24] Y. Castin, J. Phys. IV France 116, 89 (2004).

[25] E. Altman, Y. Kafri, A. Polkovnikov, and G. Refael, Phys. Rev. B 81, 174528 (2010).

[26] L. Sanchez-Palencia, Phys. Rev. A 74, 053625 (2006).

[27] D. Clément, P. Bouyer, A. Aspect, and L. Sanchez-Palencia, Phys. Rev. A 77, 033631 (2008).

[28] J. W. Goodman, Speckle Phenomena in Optics: Theory and Applications (Roberts and Company Publishers, Greenwood Village, CO, 2007). 